\title{
CHOC DES TERMES DE L'ÉCHANGE ET BALANCE COURANTE UNE ESTIMATION DES EFFETS DE SUBSTITUTION EN FRANCE
} (1972-1998)

\author{
Zoulfikar Mehoumoud Issop
}

La Documentation française | «Economie internationale »

$2001 / 2 n^{0} 86 \mid$ pages 27 à 47

ISSN 1240-8093

Article disponible en ligne à l'adresse :

https://www.cairn.info/revue-economie-internationale-2001-2-page-27.htm

\section{Pour citer cet article :}

Zoulfikar Mehoumoud Issop, " Choc des termes de l'échange et balance courante une estimation des effets de substitution en france (1972-1998) », Economie internationale 2001/2 ( $\mathrm{n}^{\circ}$ 86), p. 27-47.

Distribution électronique Cairn.info pour La Documentation française.

(c) La Documentation française. Tous droits réservés pour tous pays.

La reproduction ou représentation de cet article, notamment par photocopie, n'est autorisée que dans les limites des conditions générales d'utilisation du site ou, le cas échéant, des conditions générales de la licence souscrite par votre établissement. Toute autre reproduction ou représentation, en tout ou partie, sous quelque forme et de quelque manière que ce soit, est interdite sauf accord préalable et écrit de l'éditeur, en dehors des cas prévus par la législation en vigueur en France. Il est précisé que son stockage dans une base de données est également interdit. 


\section{CHOC DES TERMES DE L'ÉCHANGE ET BALANCE COURANTE UNE ESTIMATION DES EFFETS DE SUBSTITUTION EN FRANCE (1972-1998)}

RÉSUMÉ. L'objectif de cet article est d'évaluer les effets des chocs temporaires des termes de l'échange sur l'épargne privée et le compte courant de l'économie française. Dans un cadre intertemporel distinguant la consommation des agents selon trois types de biens (importables, exportables et non échangeables), une stratégie d'estimation en deux étapes permet d'estimer les paramètres structurels qui expliquent les fluctuations du solde courant. Les valeurs estimées des élasticités de substitution intratemporelles et intertemporelles de la consommation sont relativement faibles en France de sorte que les effets de substitution ne compensent pas les effets de lissage intertemporel de la consommation (effets revenus). Un choc temporaire défavorable et non anticipé des termes de l'échange conduit donc à une détérioration du compte courant de l'économie française.

Classification JEL: C22; D91; E21; F41; F32.

Mots-clefs : modèle intertemporel de la balance courante; élasticités de substitution ; lissage de la consommation.

Abstract. The aim of this article is to evaluate the effect of temporary terms of trade shocks on the private savings and the current account of the French economy. Using an inter-temporel framework which distinguishes agents' consumption for three types of goods (importables, exportables and non-tradables), an estimation in two stages leads to the calculation of intra-temporal and inter-temporal elasticities of substitution which explain current account fluctuations. The estimated values of these parameters are relatively low in France, so that substitution effects do not compensate inter-temporal smoothing of consumption effects. Consequently, an adverse and unanticipated terms of trade shock deteriorates the current account of the French economy.

JEL Classification: C22; D91; E21; F41; F32.

Keywords: Inter-Temporal Models of the Current Account; Elasticities of Substitution; Smoothing out of Consumption. 
$\mathrm{D}$ urant ces trente dernières années, les fortes volatilités des prix relatifs des matières premières énergétiques, des biens intermédiaires et manufacturiers ont été associées à des fluctuations importantes de l'épargne, de l'investissement et du solde courant dans les pays industrialisés. Récemment encore, les chocs sur les termes de l'échange des pays de l'UEM consécutifs à la flambée du prix du pétrole (multiplié par trois en 1999) et les dépréciations successives de l'euro, qui ont substantiellement alourdi la facture des importations, font craindre la réapparition des déséquilibres extérieurs et un ralentissement de la croissance économique européenne essentiellement tirée par la demande. Toutefois, bien que ces chocs temporaires aient des impacts directs sur les comptes extérieurs de tous les pays de la zone euro (les onze sont passés collectivement d'un excédent de 76,2 milliards d'euros, soit 1,4\% du PIB, en 1997 à un déficit courant équivalent à $0,5 \%$ du PIB au premier semestre 2000), la taille et le sens de ces effets dépendent des caractéristiques structurelles de chaque pays membre.

Traditionnellement, dans la littérature, la relation entre les termes de l'échange et la balance courante est examinée à travers deux types d'approche. Une première approche consiste à utiliser des modèles de balance commerciale de type «Mundell-Flemming » afin d'estimer des élasticités critiques (conditions de Marshall Lerner) ou de déterminer l'existence d'une courbe en J. Cependant, ces modèles puisqu'ils ne reposent pas sur des articulations (comportements) microéconomiques claires, ne prennent pas en compte la contrainte budgétaire intertemporelle de l'économie et ne précisent pas non plus la persistance des chocs. Une seconde approche consiste à utiliser les modèles intertemporels de la balance courante dans lesquels les économies échangent un même bien (ou deux biens échangeables: importables et exportables) afin de tester le comportement de lissage intertemporel de la consommation des agents. Toutefois, l'hypothèse restrictive d'un bien unique échangeable exclut les effets de substitution de la consommation sur le compte courant puisqu'elle ne tient pas compte du rôle joué, d'une part, par les transferts intertemporels de la consommation en supposant que les taux d'intérêts réels sont constants, et d'autre part, par les mouvements des taux de change réels en excluant les biens non échangeables ${ }^{2}$. Or, en général, les chocs des termes de l'échange affectent aussi bien le niveau que la composition des dépenses agrégées réelles lesquelles comprennent les non échangeables. Obstfeld et Rogoff (2000) soulignent, notamment, que l'effet de réallocation des dépenses continue d'exister dans la plupart des pays industrialisés et doit donc être au centre de toute étude de la relation entre termes de l'échange et compte courant. Au total, bien que l'approche intertemporelle à bien unique ne permette pas d'expliquer les mouvements des comptes extérieurs des pays comme le RoyaumeUni (Obstfeld \& Rogoff, 1996) ou le Canada (Gosh, 1995), elle semble être adaptée aux cas des États-Unis (Gosh, 1995) et de la France (Agénor \& alii, 1999).

L'objectif de cet article est d'évaluer les effets des chocs temporaires non anticipés des termes de l'échange sur le solde courant de l'économie française dans le cadre d'un modèle intertemporel. Les fondements microéconomiques de ce type

2. Les biens non échangeables sont définis comme ceux ne dépassant jamais les frontières nationales à cause des coûts de transports élevés ou parce que ces biens sont véritablement non échangeables (services publics, terre, logement, construction...). Or, pour beaucoup de biens, les coûts de transports (et les coûts d'échanges) sont tellement élevés qu'une proportion non négligeable du PIB des pays industrialisés peut être effectivement considérée comme non échangeable (Obstfeld, 2000). 
d'approche permettent, en effet, d'estimer les paramètres structurels de l'économie qui déterminent le sens de fluctuations du compte courant. En particulier, à l'inverse des modèles intertemporels de la balance courante avec un seul bien échangeable (largement utilisés dans la littérature), nous préférons un modèle à trois biens (importables, exportables et non échangeables) pour estimer, sur la période 1972-1998, l'ampleur des effets de substitution intertemporels (c'est-àdire les effets de substitution de la consommation présente par la consommation future) et les effets de substitution intratemporels (relatifs à la substitution entre biens importables et biens non échangeables) lors des variations transitoires des termes de l'échange en France. Des valeurs élevées de ces élasticités de substitution doivent impliquer qu'en réponse à un choc défavorable non anticipé des termes de l'échange (qui augmente le prix relatif intertemporel de la consommation et induit une appréciation temporaire réelle), la position du compte courant doit s'améliorer et compenser ainsi la détérioration induite par les effets de lissage intertemporel de la consommation.

Cet article est articulé de la manière suivante. Tout d'abord, il analyse les fondements théoriques des canaux de transmissions des chocs des termes de l'échange sur la balance courante. Puisque l'objectif principal est d'estimer l'ampleur des effets de substitution, le modèle adopté permet de donner les prédictions théoriques sur les déterminants des fluctuations du compte courant lors de chocs temporaires des termes de l'échange. La deuxième partie présente la version stochastique du modèle intertemporel estimé et la stratégie d'estimation particulière choisie. Enfin, les estimations obtenues et leurs implications pour les comptes extérieurs de l'économie française sont exposées dans la dernière partie.

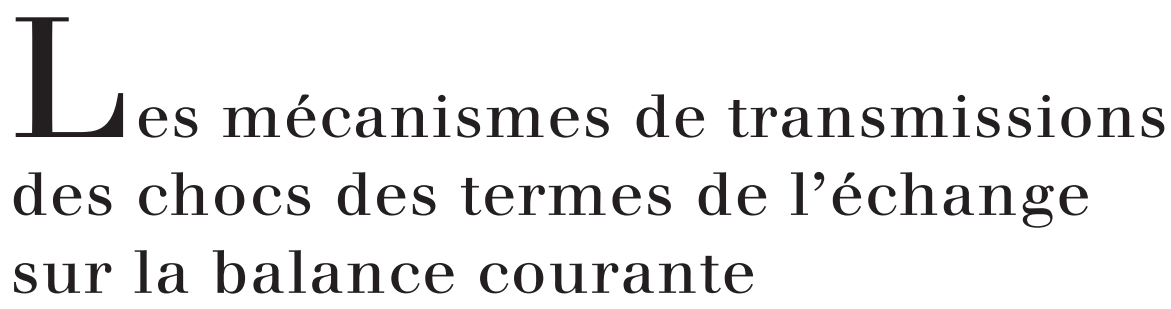

Cette partie a pour objectif de montrer le rôle des paramètres structurels de l'économie dans les mécanismes de transmission des chocs temporaires des termes de l'échange sur le compte courant à l'aide d'un modèle intertemporel déterministe simple.

\section{Le modèle intertemporel de la balance courante}

Le modèle décrit une petite économie ouverte qui produit et consomme des biens échangeables (importables) et non échangeables. L'économie considérée est une économie de dotation, on ne s'intéresse donc pas à la formation du capital. Les hypothèses sur l'environnement économique sont telles que les prévisions des agents sont parfaites, c'est-à-dire qu'ils disposent d'informations complètes et font des transactions sur des marchés parfaits (au sens où il y a intégration des marchés des biens et des capitaux).

L'analyse du comportement de l'agent représentatif permet de montrer son rôle dans les déséquilibres extérieurs. On considère que ses préférences sont 
caractérisées par la fonction d'utilité suivante qui fait entrer les niveaux de consommations de manière additive à travers le temps :

$U_{t}=\sum_{s=t}^{\infty} \beta^{s-t} \frac{C_{s}^{1-\frac{1}{\sigma}}}{1-\frac{1}{\sigma}}$

où $\beta \in[0,1]$ représente le paramètre de préférence pour le présent de l'agent représentatif. $C_{s}$ est le panier de consommation défini plus bas et $\sigma>0$ est l'élasticité de substitution intertemporelle qui mesure la sensibilité de l'allocation intertemporelle de la consommation à une variation de taux d'intérêt réel. Puisque l'objectif de l'agent consiste aussi bien à rechercher sa consommation optimale dans le temps que la demande des différents types de biens, il est nécessaire d'analyser respectivement ses choix intratemporels et intertemporels.

LE PROBLÈME INTRATEMPOREL DE L'AGENT REPRÉSENTATIF. Comme l'agent représentatif consomme à la fois des biens importables et non échangeables, on suppose que sa fonction de consommation est représentée par un indice composite CES («Constant Elasticity of Substitution »):

$C_{t}=\left[w m_{t}^{1-\frac{1}{\theta}}+n_{t}^{1-\frac{1}{\theta}}\right]^{\frac{\theta}{\theta-1}}$

où $m_{t}$ et $n_{t}$ représentent respectivement la consommation de biens importables et non échangeables et $w$ est le poids relatif des biens importables dans la consommation. Les biens exportables produits par l'économie domestique sont supposés ne pas être utilisés dans la consommation locale ${ }^{3} . \theta>0$ est l'élasticité de substitution intratemporelle entre biens importables et biens non échangeables. Elle mesure le degré avec lequel l'agent substitue sa consommation de bien importable en faveur de celle de bien non échangeable produit par l'économie domestique lors d'une variation du prix relatif des biens importables par rapport à celle des biens non échangeables. Une valeur de $\theta$ supérieure (inférieure) à l'unité implique que les biens non échangeables et importables sont substituables (complémentaires). On notera que la décomposition du panier de consommation de l'agent qui est retenue ici est différente de celle traditionnellement retenue dans un modèle avec bien échangeable et non échangeable (Obstfeld \& Rogoff, 1994 et 1996). En effet, dans ce type d'approche, les prix relatifs des biens importables et exportables (les termes de l'échange) sont implicitement considérés comme constants de sorte que les deux biens peuvent être agrégés en un seul bien échangeable homogène. Or, cette agrégation peut biaiser les résultats empiriques du modèle lorsque les termes de l'échange fluctuent significativement dans le temps.

3. Bien que cette hypothèse soit assez simplificatrice dans le cas de la France, ce choix de spécification de la consommation a été adopté afin de rendre le modèle empiriquement utile. Les donnés disponibles ne permettent pas une désagrégation plus fine de la consommation entre biens exportables et importables. De plus, ce choix n'a pas d'influence sur l'analyse théorique puisque les mécanismes de transmission des chocs des termes de l'échange sur le solde courant ne se trouvent pas modifiés lorsqu'on incorpore les biens exportables dans la consommation (voir Ostry, 1988 et Ostry \& Reinhardt, 1992). 
Soit $Z_{t}$ le niveau de dépenses de consommation de l'agent, exprimé en termes de biens exportables, par:

$Z_{t}=p_{t} m_{t}+q_{t} n_{t}$

où $p_{t}$ est le prix relatif du bien importable (en fait, les termes de l'échange ${ }^{4}$ ) et $q_{t}$ est le prix relatif du bien non échangeable (ou l'inverse du taux de change réel ${ }^{5}$ ).

La maximisation intratemporelle de (2) sous la contrainte (3) doit alors vérifier la condition de premier ordre telle que le taux marginal de substitution intratemporel entre les biens importables et les biens non échangeables soit égal à leur prix relatif à long terme :

$w\left(\frac{n_{t}}{m_{t}}\right)^{\frac{1}{\theta}}=\frac{p_{t}}{q_{t}}$

L'indice général des prix à la consommation $P_{t}$, en termes de biens exportables, s'obtient alors comme le coût minimum payé par l'agent pour se procurer une unité du panier de biens $C_{t}$ :

$P_{t}=\left[w^{\theta} p_{t}^{1-\theta}+q_{t}^{1-\theta}\right]^{\frac{1}{1-\theta}}$

Puisque $Z_{t} / P_{t}=C_{t}$ est le ratio de dépense d'une unité de consommation, l'indice des prix obtenu permet ainsi de traduire les dépenses de consommation en termes de consommation réelle $C_{t}$. En outre, l'indice des prix $P_{t}$ varie dans le même sens que les termes de l'échange et le taux de change réel $\left(\frac{\Delta P_{t}}{\Delta p_{t}}>0 ; \frac{\Delta P_{t}}{\Delta q_{t}}>0\right)$. Enfin, les demandes optimales de biens, proportionnelles à la consommation réelle $C_{t}$ selon une fonction isoélastique du prix relatif du bien, sont données par:

$m_{t}=w^{\theta}\left(\frac{p_{t}}{P_{t}}\right)^{-\theta} C_{t}$

$n_{t}=\left(\frac{q_{t}}{P_{t}}\right)^{-\theta} C_{t}$

Le programme intertemporel de L'Agent. Pour déterminer maintenant le choix intertemporel optimal de l'agent, il est tout d'abord nécessaire d'établir sa contrainte budgétaire intertemporelle (CBI). On suppose qu'il n'y a pas de dépenses publiques, ni d'investissement. L'accumulation nette d'actif

4. Les termes de l'échange sont définis ici comme le prix relatif des biens importés sur les prix des biens exportés. D'où, une détérioration des termes de l'échange est équivalente à une hausse du prix relatif des biens importés puisque d'après la mesure usuelle des termes de l'échange, celle-ci est égale aux prix des exportations sur les prix des importations.

5. Le taux de change réel est égal à l'inverse du prix relatif des biens non échangeables puisque si on suppose que la PPA (Parité de Pouvoir d'Achat) est respectée, l'égalisation des prix des biens échangeables entre les pays permet d'expliquer l'évolution du taux de change réel uniquement en termes de prix des biens non échangeables (Obstfeld \& Rogoff, 1996). 
par l'agent, le compte courant, sur une infinité de périodes représente la CBI de l'économie et compte tenu de la présence des biens non échangeables, elle est définie en termes de biens exportables par:

$\sum_{s=t}^{\infty} R_{t, s} P_{s} C_{s}=\left(1+r_{t}\right) B_{t}+\sum_{s=t}^{\infty} R_{t, s} P_{s} Y_{s}$

où, $R_{t, s}=\frac{1}{\prod_{v=t+1}^{s}\left(1+r_{v}\right)}$ est le coefficient d'actualisation de l'agrégat en $s$ à la date $t$ et où $r_{t}$ est le taux d'intérêt mondial entre $t$ et $t-1 . B_{t}$ est le stock d'avoirs nets à l'étranger à la fin de la période $t-1 . P_{s} C_{s}=p_{s} m_{s}+q_{s} n_{s}$ représente la valeur du panier de biens de consommation de l'agent en termes de bien exportables. $P_{s} Y_{s}=Y_{s}^{T}+q_{s} Y_{s}^{n}$ est la valeur de la production domestique exprimée en terme de biens exportables. Enfin, $Y_{s}^{T}=p_{s} \bar{m}_{s}+x_{s}$ représente la valeur de la dotation de biens échangeables en terme de biens exportables; $\bar{m}_{s}$ étant la dotation de biens importables (ou encore la production des substituts aux importations), et $x_{s}$ est la production de biens exportés au reste du monde ${ }^{6}$.

La CBI (8) établit que la valeur présente des dépenses d'une économie doit être égale à sa richesse financière initiale plus la valeur actualisée de la production domestique. À l'équilibre général, la vérification de l'équilibre extérieur suppose le respect de la CBI. Toutefois, les circonstances de déséquilibre de la balance courante vont surgir du comportement de lissage intertemporel de consommation de l'agent suite à une variation de son revenu, ainsi que de celui de substitution intertemporel et intratemporel de la consommation des biens suite à une variation des prix relatifs (termes de l'échange et taux de change réel).

Les conditions d'optimalité intertemporelles de la maximisation de (1) sous la CBI (8) permettent d'obtenir ${ }^{7}$ la relation d'Euler suivante:

$\beta\left(\frac{C_{t+1}}{C_{t}}\right)^{-\frac{1}{\sigma}}=\frac{1}{1+r_{t+1}} \frac{P_{t+1}}{P_{t}}$

La relation d'Euler représente tout simplement l'égalisation entre le taux marginal de substitution intertemporel et le prix relatif intertemporel. Le facteur d'intérêt $\frac{1}{1+r_{t+1}}$, qui est le prix intertemporel du bien échangeable, ajusté par $\frac{P_{t+1}}{P_{t}}$, n'est alors rien d'autre que le taux d'intérêt réel du panier de bien de consommation d'importables et de non échangeables. Ce dernier est donc le prix intertemporel d'une unité de consommation réelle, c'est-à-dire, le prix de la consommation future du panier de biens en terme de consommation présente.

6. Par hypothèse, la production des substituts aux importations est entièrement consommée localement alors que la production des biens exportables est entièrement destinée aux marchés étrangers.

7. Sous respect de la condition de «transversalité »: $\lim _{s \rightarrow \infty} R_{t, s} B_{s}=0$. 


\section{Les motifs de fluctuations du compte courant}

Il est maintenant possible de déterminer la dynamique du compte courant et d'étudier les motifs de fluctuations de ce dernier à partir des conditions d'équilibre intratemporelles et intertemporelles de l'agent obtenues précédemment.

La Dynamique Du compte courant. Compte tenu de la demande optimale intratemporelle (6), la condition d'Euler pour les biens importables, qui doit être vérifiée pour toutes périodes $s$ (quel que soit $s>t$ ), est:

$m_{s}=\beta^{(s-t) \sigma} R_{t, s}^{-\sigma}\left(\frac{P_{t}}{P_{s}}\right)^{\sigma-\theta}\left(\frac{p_{t}}{p_{s}}\right)^{\theta} m_{t}$

En supposant que le marché des biens non échangeables est en équilibre $\left(n_{s}=Y_{s}^{n}\right)$ et en incorporant (10) dans (8), la consommation optimale des biens importables est alors:

$$
m_{t}=\frac{\frac{P_{t}}{p_{t}}\left[\left(1+r_{t}\right) B_{t}+\sum_{s=t}^{\infty} R_{t, s} \frac{Y_{s}^{T}}{P_{s}}\right]}{\sum_{s=t}^{\infty} R_{t, s}\left(\frac{\beta^{s-t}}{R_{t, s}}\right)^{\sigma}\left(\frac{P_{t}}{P_{s}}\right)^{\sigma}\left(\frac{p_{t} / P_{t}}{p_{s} / P_{s}}\right)^{\theta-1}}
$$

Puisque le compte courant reflète l'échange de production nette entre les économies, il doit être mesuré en terme de bien échangeables. À l'équilibre, la différence entre le revenu et les dépenses sur les biens échangeables (en terme réel) s'écrit alors :

$\frac{C A_{t}}{P_{t}}=\frac{B_{t+1}-B_{t}}{P_{t}}=r_{t} \frac{B_{t}}{P_{t}}+\frac{Y_{t}^{T}}{P_{t}}-\frac{p_{t}}{P_{t}} m_{t}$

$\mathrm{Si}$ on définit le niveau permanent d'une variable $X_{t}$ par $\sum_{s=t}^{\infty} R_{t, s} X_{s}=\sum_{s=t}^{\infty} R_{t, s} \tilde{X}_{t}$ et en incorporant (11) dans (12), on obtient alors l'équation fondamentale suivante qui décrit la dynamique réelle du compte courant:

$$
\begin{aligned}
\frac{C A_{t}}{P_{t}}=\left(r_{t}-\tilde{r}_{t}\right) \frac{B_{t}}{P_{t}}+\left(\frac{Y_{t}^{T}}{P_{t}}-\frac{\tilde{Y}_{t}^{T}}{P_{t}}\right)+\underbrace{\left(\tilde{r}_{t} \frac{B_{t}}{P_{t}}+\frac{\tilde{Y}_{t}^{T}}{P_{t}}\right)}_{\text {ressources permanentes }}\left[1-\frac{1}{\Delta}\right] \\
\text { où } \Delta=\frac{\sum_{s=t}^{\infty} R_{t, s}\left(\frac{\beta^{s-t}}{R_{t, s}}\right)^{\sigma}\left(\frac{P_{t}}{P_{s}}\right)^{\sigma}\left(\frac{p_{t} / P_{t}}{p_{s} / P_{s}}\right)^{\theta-1}}{\sum_{s=t}^{\infty} R_{t, s}}
\end{aligned}
$$


L'équation (13) permet alors de mettre en évidence les effets de lissage et de substitution intertemporelle et intratemporelle de la consommation des agents sur le compte courant.

LES EFFETS DES CHOCS DE TERMES DE L'ÉCHANGE SUR LE COMPTE COURANT. Le premier motif de fluctuations du solde courant qui apparaît est dû à l'effet de lissage intertemporel de la consommation. C'est l'influence de l'effet d'amortissement dans le temps des fluctuations de la consommation sur le compte courant. En effet, lorsque la production réelle (ou le revenu réel) est au-dessus de sa valeur permanente $\left(d \frac{Y_{t}^{T}}{P_{t}}>d \frac{\tilde{Y}_{t}^{T}}{P_{t}}\right)$, le compte courant sera excédentaire car les agents étaleront dans le temps ce gain transitoire de revenu en augmentant leur épargne. Au lieu d'augmenter leur consommation courante, les agents choisissent d'accumuler des actifs financiers renumérateurs d'intérêts pour lisser leur consommation sur les périodes futures ${ }^{8}$. Cet effet de lissage intertemporel de la consommation permet ainsi de mettre en évidence l'explication traditionnelle de l'effet revenu des variations temporaires des termes de l'échange sur le compte courant (Harberger-Laurzen-Metzler, 1950). Ainsi, une détérioration temporaire des termes de l'échange d'un pays provoque une baisse transitoire de son revenu réel courant relativement à son revenu réel permanent (la baisse du revenu réel permanent est donc plus faible que celle du revenu courant ${ }^{9}$ ). Du fait du comportement de lissage de la consommation, cette baisse temporaire de revenu sera amortie par une réduction de l'épargne (ou par une hausse des emprunts) puisque la baisse de la consommation présente est plus faible que celle du revenu réel courant. On assiste alors à une détérioration du compte courant. Il faut noter que cet effet revenu est vérifié uniquement lorsque les variations des termes de l'échange sont transitoires (Obstfeld, 1982; Gavin, 1990). Par exemple, si les agents anticipent une amélioration permanente des termes de l'échange, ils vont réviser à la hausse leur niveau de consommation courant dans le même ordre que la hausse du revenu réel permanent, ce qui n'a aucun effet sur la dynamique du compte courant.

À côté de cet effet quantité, les variations des termes de l'échange entraînent également des effets substitution sur le compte courant liés à la modification des prix relatifs. Ces effets peuvent compenser partiellement ou totalement l'effet de lissage intertemporel de la consommation. Plus précisément, deux types d'effets de prix relatifs sont mis en évidence à partir du paramètre $\Delta$ de l'équation (13).

Le premier effet de substitution est un effet de transfert intertemporel de la consommation proportionnel à l'élasticité de substitution intertemporelle $\sigma$. Il est lié à la réallocation de la consommation dans le temps suite à la variation de l'indice des prix à la consommation. Une détérioration temporaire des termes l'échange $\left(p_{t}>p_{s}\right)$ entraîne, en effet, une augmentation temporaire de l'indice

8. L'effet de lissage intertemporel de la consommation va aussi se manifester lors des variations temporaires de taux d'intérêt. En effet, lorsque l'économie est créditrice nette vis-à-vis du reste du monde, toute variation du taux d'intérêt audessus de sa valeur provoquera des excédents de la balance courante. Et inversement, lorsque l'économie est débitrice nette vis-à-vis du reste du monde, des hausses temporaires des taux provoqueront des déficits courants.

9. La réduction du revenu réel s'explique par la baisse du pouvoir d'achat de la production des exportables. 
général des prix $\left(P_{t}>P_{s}\right)$. La hausse du taux d'intérêt réel de la consommation qui en résulte implique que le prix de la consommation présente de biens importés sera relativement plus élevé que celui de la consommation future. Les agents transfèrent dans ce cas une partie de la consommation dans le temps en augmentant leur épargne. Ce qui se traduit par une amélioration du compte courant. Plus $\sigma$ est élevé, plus les agents tendent à réajuster leur consommation en réponse aux mouvements des prix relatifs intertemporels.

Le second effet de substitution est un effet de taux de change réel proportionnel à l'élasticité de substitution intratemporelle $\theta$. Il est lié à la modification de la répartition de la consommation entre les biens échangeables et les biens domestiques à la suite de la modification du prix du bien importable relativement à la variation de l'indice global des prix. Dans le cas d'une détérioration temporaire des termes de l'échange (c'est-à-dire une hausse du prix relatif des biens importables relativement à l'indice général des prix), les agents tendent à accroître la part des biens non échangeables dans leur consommation. Dans le même temps, l'accroissement de la consommation des biens non échangeables, sous l'hypothèse que le marché des biens non échangeables est en équilibre (c'est-à-dire qu'il n'y existe pas de ressources sous-exploitées dans l'économie domestique), augmente leur prix relatif qui n'est rien d'autre que le taux de change réel. L'appréciation réelle temporaire provoque alors une augmentation transitoire du niveau général des prix, nouvelle source de substitution intertemporelle. Cet effet de substitution s'accompagne dans ce cas d'excédents courants jusqu'à ce que le prix des biens importables revienne à son niveau permanent. Inversement, lors d'une amélioration des termes de l'échange, la substitution des dépenses de consommation vers les biens importables et la baisse temporaire de l'indice des prix s'accompagnent d'une diminution du taux d'intérêt réel de la consommation qui entraîne des déficits courants puisque les agents vont avoir une préférence biaisée vers la consommation présente. Enfin, la substitution de la consommation vers les biens non échangeables, l'appréciation du taux de change réel et l'augmentation du taux d'intérêt réel de la consommation seront d'autant plus fortes, lors d'une détérioration temporaire des termes de l'échange, que la valeur de $\theta$ est élevée.

Il est ainsi clair que ces effets de substitution intertemporelle et intratemporelle sont essentiels pour expliquer les mouvements du compte courant de l'économie face aux chocs de prix relatifs. Suite à une variation temporaire des termes de l'échange, le sens de fluctuation du compte courant dû aux motifs de transfert intertemporel et de taux de change réel est complètement à l'opposé de celui dû au motif de lissage de consommation. Après une détérioration des termes de l'échange, plus les élasticités intertemporelles et intratemporelles sont élevées, plus faible est la détérioration du solde courant induite par le motif de lissage intertemporelle de la consommation. Une évaluation de la taille relative de ces différents effets permet donc de comprendre la nature et l'ampleur des mécanismes macroéconomiques à l'œuvre dans la détermination des fluctuations de la balance courante lors d'une modification temporaire non anticipé des termes de l'échange. Cette évaluation passe ici par une estimation des paramètres structurels du modèle théorique précédemment exposé. 


\section{Modèle estimé et stratégies d'estimation}

Le vecteur des paramètres structurels $\{w, \theta, \beta, \sigma\}$ du modèle est ici estimé à partir des conditions de premier ordre du programme de l'agent représentatif. L'estimation passe cependant par la réécriture du modèle de choix de l'agent représentatif dans un environnement stochastique. La stratégie d'estimation choisie est ensuite basée sur l'approche en deux étapes, similaires à celle proposée par Ostry et Reinhardt (1992), Cooley et Ogaki (1996) et Cashin et Mc Dermott (1998).

\section{La version stochastique du modèle}

Dans un cadre d'environnement incertain, on suppose que les préférences de l'agent sont caractérisées par la fonction d'utilité espérée suivante:

$U_{t}=\left(\frac{\sigma}{\sigma-1}\right) E_{t} \sum_{t=0}^{\infty} \beta_{t}\left[w m_{t}^{1-\frac{1}{\theta}}+n_{t}{ }^{1-\frac{1}{\theta}}\right]^{\frac{1-1 / \sigma}{1-1 / \theta}}$

La solution du programme de maximisation de cette utilité est donnée par la séquence $\left\{m_{t}, m_{t+1}, n_{t}, n_{t+1}\right\}$ qui maximise (14) sous la CBI (8). Les conditions de premier ordre donnent les équations stochastiques d'Euler et de long terme suivantes:

$$
\begin{aligned}
& E_{t}\left\{\left(1+r_{t}\right) \frac{p_{t}}{p_{t+1}}\left[\frac{w m_{t+1}^{1-1 / \theta}+n_{t+1}^{1-1 / \theta}}{w m_{t}^{1-1 / \theta}+n_{t}^{1-1 / \theta}}\right]^{\frac{\sigma-\theta}{\sigma(\theta-1)}}\left[\frac{m_{t+1}}{m_{t}}\right]^{-\frac{1}{\theta}}\right\}=\frac{1}{\beta} \\
& E_{t}\left\{\left(1+r_{t}\right) \frac{q_{t}}{q_{t+1}}\left[\frac{w m_{t+1}^{1-1 / \theta}+n_{t+1}^{1-1 / \theta}}{w m_{t}^{1-1 / \theta}+n_{t}^{1-1 / \theta}}\right]^{\frac{\sigma-\theta}{\sigma(\theta-1)}}\left[\frac{n_{t+1}}{n_{t}}\right]^{-\frac{1}{\theta}}\right\}=\frac{1}{\beta} \\
& w\left(\frac{n_{t}}{m_{t}}\right)^{\frac{1}{\theta}}=\frac{p_{t}}{q_{t}}
\end{aligned}
$$

Ces équations sont comparables à celles obtenues dans un environnement certain. L'équation (17) est comparable à celle trouvée dans le programme de maximisation intratemporel de l'agent telle que le taux marginal de substitution intratemporel est égal au rapport des prix relatifs à long terme. Puisque les équations (15), (16) et (17) ne sont pas indépendantes ${ }^{10}$, l'estimation de deux équations uniquement suffit ici pour estimer les paramètres structurels. 


\section{Une méthode d'estimation en deux étapes}

La stratégie d'estimation consiste, dans une première étape, à estimer les paramètres de préférence $w$ et $\theta$ à partir d'une régression de cointégration de la relation de long terme (17) et, dans une deuxième étape, à imposer les valeurs estimées de ses paramètres dans l'équation d'Euler (15) pour estimer les paramètres restants $\beta$ et $\sigma$ par la méthode des moments généralisés (GMM) de Hansen (1982). Cette procédure à deux étapes n'altère pas la distribution asymptotique des estimateurs GMM et des tests usuels car les estimateurs $w$ et $\theta$ obtenus par la régression de cointégration sont super-consistents et convergent à un taux supérieur à $T^{1 / 2}$. Pour améliorer l'efficacité des paramètres estimés à la première étape, nous avons estimé la régression (17) par les Moindres Carrés Ordinaires Dynamiques (MCOD). En effet, dans la mesure où l'estimation par la méthode des MCO peut souffrir de biais d'échantillonnage "1, dans le cas d'un échantillon de petite taille comme nous avons eu à faire face, la méthode des MCOD de Stock et Watson (1993) permet de corriger ce biais et de retomber sur l'inférence économétrique standard (Hamilton, 1994). Le principe des MCOD consiste à ajouter des décalages passés et futurs à la différence première de la variable de droite de la régression de cointégration:

$$
\ln \left(\frac{p_{t}}{q_{t}}\right)=\ln (\hat{w})+\frac{1}{\hat{\theta}} \ln \left(\frac{n_{t}}{m_{t}}\right)+\sum_{j=-k}^{k} \hat{\lambda}_{j} \Delta \ln \left(\frac{n_{t+j}}{m_{t+j}}\right)+\hat{e}_{t}
$$

Concernant l'estimation par la méthode des GMM, les résidus de la condition d'Euler (15) peuvent être définis par:

$$
u_{t}=\left\{\left(1+r_{t}\right) \frac{p_{t}}{p_{t+1}}\left[\frac{w m_{t+1}^{1-1 / \theta}+n_{t+1}^{1-1 / \theta}}{w m_{t}^{1-1 / \theta}+n_{t}^{1-1 / \theta}}\right]^{\frac{\sigma-\theta}{\sigma(\theta-1)}}\left[\frac{m_{t+1}}{m_{t}}\right]^{-\frac{1}{\theta}}\right\}-\frac{1}{\beta}
$$

Sous l'hypothèse d'anticipations rationnelles, les résidus sont non corrélés avec les informations dont disposent les agents à l'instant $t$. Ces erreurs de prévisions sont donc orthogonales à tout instrument connu par l'agent à l'instant $t$. Ainsi, l'hypothèse d'anticipation rationnelle selon laquelle les agents utilisent toutes les informations disponibles pour prévoir les prix relatifs et les consommations futures nous permet d'utiliser un nombre important de variables instrumentales pour estimer un petit nombre de paramètres. De plus, les termes d'erreurs pouvant inclure des erreurs de mesure sur les données, il nous faut donc choisir des instruments pertinents de telle sorte qu'ils ne soient pas corrélés avec les erreurs.

Il est alors possible d'imposer les conditions d'orthogonalité (ou conditions de moments) suivantes: $E\left(u_{t} Z_{t}\right)=0$ où $Z_{t}$ est ici le vecteur de six variables instrumentales ${ }^{12}$ défini par: $Z_{t}^{\prime}=\left[\right.$ constante, $\left.\frac{m_{t-1}}{m_{t-2}}, \frac{n_{t-1}}{n_{t-2}},\left(1+r_{t-2}\right) \frac{p_{t-2}}{p_{t-1}}, m_{t-1}, n_{t-1}\right]$.

11. L'ordre du biais est de $T^{-1}$ et la source du biais provient du biais de simultanéité (corrélation entre variable explicative et termes d'erreurs) et de l'autocorrélation des résidus (Hamilton, 1994).

12. Notons qu'aucune variable instrumentale choisie n'est considérée à la date $t$ car le processus de moyenne mobile des termes d'erreurs implique une corrélation de ces variables avec les résidus $u_{t}$. 
L'estimation GMM de $\beta$ et $\sigma$ est alors obtenue en minimisant la fonction $J$ suivante (Ogaki, 1993):

$J=m^{\prime} W m$

où $m=\frac{1}{T} Z^{\prime} u$ sont les moments d'échantillon et $W$ est une matrice semi-definie positive (encore appelée, matrice distance). Puisque le nombre de conditions d'orthogonalité (six) est supérieur au nombre de paramètres à estimer (deux), le système est ici suridentifié. Différents estimateurs GMM peuvent être obtenus en fonction du choix de la matrice distance $W$ lorsqu'il y a des restrictions de suridentification. Hansen (1982) montre alors qu'on peut obtenir des estimateurs GMM efficaces (étant donné les variables instrumentales) en choisissant pour cette matrice l'inverse de la matrice de variance covariance des moments d'échantillon. Enfin, pour tester la validité des conditions des moments imposées, nous utilisons le test $J$ proposé par Hansen (1982). Ce test est ici retenu pour tester l'hypothèse nulle selon laquelle les quatre restrictions de suridentifications ne sont pas rejetées par les données et les paramètres estimés sont efficients. La valeur minimum de la fonction objectif, $J$, suit ici une loi de $\chi^{2}$ avec quatre degrés de liberté.

\section{L'importance des effets de lissage (revenus) dans la détermination du compte courant en France}

Cette procédure d'estimation a ensuite été appliquée à l'économie française ${ }^{13}$ sur la période 1972-1998, en données annuelles. Les séries sur la consommation des biens importables et des biens non échangeables en France ont été construites grâce à la méthodologie ${ }^{14}$ proposée par Ostry et Reinhardt (1992) et Cashin et Mc Dermott (1998).

Les différents tests de racine unitaire et de cointégration (TABLEAU A.2.1 en ANNEXE A.2) indiquent que $\frac{n_{t}}{m_{t}}$ et $\frac{p_{t}}{q_{t}}$ sont I (1) (aussi bien en niveau qu'en logarithme) et cointégrés. La statistique du test $J$ est inférieure à sa valeur critique (TABLEAU 1). Les restrictions de sur-identification imposées à notre modèle non linéaire ne sont donc pas rejetées par les données au seuil de $5 \%$. Les six conditions d'orthogonalité donnent par conséquent des estimations GMM efficaces des deux paramètres $\beta$ et $\sigma$. Enfin, l'estimation du paramètre de préférence pour le présent (facteur d'actualisation subjectif) est significativement différent de zéro et est bien identifié à $0,899(<1)$ puisque l'hypothèse que celui ci soit égal à un est rejeté au seuil de $5 \%$. Cela implique que les agents économiques français ont une

13. Un travail préalable nous a permis d'établir que les variations des termes de l'échange sont de nature typiquement temporaire en France. En effet, grâce à l'indicateur de persistance des chocs de Cochrane (1988), nous montrons que la part de la composante permanente des mouvements des termes de l'échange est relativement faible (de $45 \%$ ) au bout de 20 trimestres. Les résultats de ce travail sont disponibles auprès de l'auteur.

14. Voir en ANNEXE A.1 la présentation des sources et la construction des séries. 
TABleau 1

\begin{tabular}{|ccccc|}
\hline & & \multicolumn{2}{c|}{ Résultats des estimations* } \\
$w$ & $\theta$ & $\beta$ & $\sigma$ & $J$ \\
0,271 & 0,745 & 0,899 & 1,52 & 5,84 \\
$(0,272)$ & $(0,03)$ & $(0,858)$ & $(0,211)$ \\
* Les valeurs entre parenthèses sont les écarts-type des paramètres estimés et la «p-value » de la statistique du test J. Pour les esti- \\
mations MCOD, un nombre de décalages k = 3 a été retenu.
\end{tabular}

préférence relativement plus forte pour le présent, c'est-à-dire qu'ils sont relativement impatients à consommer plus aujourd'hui qu'épargner.

\section{Estimation de l'élasticité de substitution intertemporelle}

La valeur estimée de l'élasticité de substitution intertemporelle $\sigma$ est élevée mais n'est pas statistiquement significative. Lorsqu' on compare les valeurs de $\sigma$ obtenues par Cashin et Mc Dermott (1998) dans le cas des pays développés, et par Ostry et Reihardt (1992) pour les pays en voie de développement, ces dernières sont plus élevées pour les pays riches (TABLEAU A.2.2 en ANNEXE A.2). On peut raisonnablement penser alors qu'en France, les dépenses de consommation des agents en biens fortement élastiques sont beaucoup plus substituables dans le temps que celles des pays en voie de développement qui concernent essentiellement des biens inélastiques (tels que les biens de subsistance et de première nécessité). Toutefois, Cashin et Mc Dermott (1998) trouvent, pour l'Australie, les États-Unis, le Canada et le Royaume-Uni, des valeurs de $\sigma$ significativement différentes de zéro indiquant pour ses pays des effets de substitutions intertemporels et donc des transferts de consommation élevés qui tendent à compenser les effets de lissage (TABLEAU A.2.2).

À l'opposé, l'estimation de la valeur de $\sigma$ semble rejoindre les résultats obtenus par Agénor et alii (1999) pour la France. Ces auteurs utilisent la méthodologie de Gosh (1995) pour calculer le paramètre de transfert intertemporel de consommation dans un modèle à bien unique avec une fonction d'utilité instantanée quadratique (permettant ainsi d'éliminer le paramètre d'élasticité de substitution intertemporel). Ils estiment, en fait, une relation de cointégration entre le « cash-flow » national (défini comme la production nette des investissements et des dépenses publiques) et la consommation privée. Ils obtiennent ainsi, sur la période 1970:1-1996:4, une estimation du paramètre de transfert intertemporel de l'ordre de 0,982 statistiquement différent de 1 indiquant que la France a consommé plus que son cash-flow permanent et a eu une tendance prononcée pour la consommation présente sur cette période du fait d'un profil de consommation biaisé vers le présent. Ce résultat est compatible avec notre estimation d'une élasticité de substitution non significative dans la mesure où elle indique un transfert de consommation faible entre les périodes.

Une valeur de $\sigma$ non statistiquement différente de zéro a été généralement trouvée dans la littérature sur des estimations des équations d'Euler linéarisées dans le cadre d'un modèle à bien unique (Obstfeld \& Rogoff, 1996; Cashin \& Mc Dermott, 1998). Nous avons alors reproduit la version stochastique du modèle de 
revenu permanent à bien unique pour vérifier la valeur de l'élasticité intertemporelle français dans ce cadre:

$\Delta C_{t}=\mu+\sigma^{\prime} R_{t}+\varepsilon_{t}$

où $\Delta C_{t}$ représente la différence première du logarithme de la consommation agrégée (la somme de la consommation des biens non échangeables et importables), $R_{t}$ est le taux d'intérêt réel, $\mu$, une constante et enfin, $\sigma$ 'représente l'élasticité de substitution intertemporelle dans ce type de modèle.

En estimant (21) par la méthode des variables instrumentales linéaires (afin de tenir compte de la corrélation des termes d'erreurs avec les variables explicatives), nous retrouvons une relation statistiquement non significative entre le taux d'intérêt réel et la variation de la consommation à un seuil critique de $5 \%$ (TABLEAU 2). Toutefois, sa valeur est proche de zéro et est relativement plus faible que l'estimation trouvée dans le modèle à deux biens. L'estimation de $\sigma$ dérivée d'un modèle à bien unique est généralement considérée dans la littérature comme inappropriée pour des pays qui sont sujets à des chocs fréquents de leurs termes de l'échange puisque ces derniers altèrent de manière importante leur taux de change réel et font donc varier les prix relatifs des biens importables et non échangeables. L'hypothèse d'un modèle à bien unique apparaît comme valide pour la France moins sujette à des chocs importants de ses termes de l'échange. Pour autant, les estimations dans un modèle de consommation avec biens échangeables et non échangeables permettent de tenir compte des influences du taux d'intérêt réel sur la consommation des différents biens et ne biaisent pas ainsi la valeur de $\sigma$ vers zéro.

TABLEAU 2

Résultats de l'estimation de l'équation linéaire*

Paramètres

$\mu$

$\sigma$
Valeurs estimées

0,04

$(0,02)$

0,013

$(0,008)$

Les variables instrumentales sont $R_{t-2}, \Delta C_{t-2}$ et $\Delta \ln \left(d e f_{t-2}\right)$.

Les valeurs entre parenthèses sont les écarts-type des paramètres estimés.

\section{Estimation de l'élasticité de substitution intratemporelle}

Les valeurs estimées des paramètres $w$ et $\theta$ par les MCOD sont présentées dans le TABLEAU 1. Les coefficients associés aux variables en différences premières ne sont pas présentés. La valeur estimée de $\theta$ est relativement faible, de l'ordre de 0,745. Les différents tests de Wald menés sur les estimations MCOD (TABLEAU 3) aboutissent aux résultats suivants :

— l'hypothèse nulle selon laquelle le paramètre $\theta$ n'est pas significatif peut être rejetée au seuil de $5 \%$;

— l'hypothèse selon laquelle celui ci est égal à un est également rejetée ; 
- la méthode MCOD de Stock et Watson (1993) donne des estimateurs plus efficients que celle des MCO classiques puisque les tests de significativité des coefficients en décalages passés et futurs de la variable explicative montrent que ces derniers sont significativement différents de zéro.

Bien que les MCOD donnent des paramètres plus efficients et plus élevés que les MCO classiques, l'hypothèse que les biens importables et non échangeables soient complémentaires ou imparfaitement substituables $(\theta<1)$ en France ne peut être rejetée. En d'autres termes, lors d'une détérioration transitoire des termes de l'échange, les agents privés français ne substituent pas parfaitement les biens importés qui sont relativement plus chers en faveur des biens domestiques (non échangeables). La faiblesse de l'élasticité de substitution intratemporelle implique que l'appréciation du taux de change réel et l'augmentation du taux d'intérêt réel de la consommation sont faibles. La hausse de l'épargne privée qui s'ensuit n'est pas suffisante pour compenser totalement la réduction de celle-ci due à l'effet de lissage intertemporel de la consommation.

TABLEAU 3

Tests de Wald*

Hypothèse nulle

$\begin{array}{ccc}\theta=1 & \theta=0 & \lambda_{j}=0 \\ 8,727 & 134,66 & 131,52 \\ (0,003) & (0,000) & (0,000)\end{array}$

* Les statistiques de Wald suivent une loi du $\chi^{2}(n)$ sous l'hypothèse nulle où $n$ est le nombre de paramètres estimés. Les valeurs entre parenthèses sont les «p-value».

Au total, le compte courant va donc se détériorer puisque les effets de substitution intertemporels (dont l'élasticité n'est pas significativement différente de zéro) et intratemporels $(\theta<1)$ ne contrebalancent pas les effets revenus induits par la détérioration transitoire des termes de l'échange. Ce résultat est ici différent de celui trouvé par Cashin et Mc Dermott (1998) pour l'Australie, le Canada et le Royaume-Uni où les effets de transferts intertemporels de consommation $(\sigma$ significativement élevé et différent de zéro) et de taux de change réel $(\theta>1)$ compensent les variations du solde courant induites par l'effet de lissage intertemporel de la consommation (TABLEAU A.2.2). Cette divergence des paramètres structurels est sans doute liée à la plus grande sensibilité du commerce extérieur de ces trois pays aux variations des termes de l'échange, compte tenu du poids important des matières premières dans leurs exportations (Cashin \& Mc Dermott, 1998).

Le réexamen sur le passé de la corrélation entre variation des termes de l'échange et compte courant, et la simulation de la taille relative des différents effets, compte tenu des valeurs estimées des paramètres structurels, confirment les résultats empiriques obtenus. En effet, d'après le nuage de points du GRAPHIQUE 1, les chocs des termes de l'échange et la balance courante en France sont négativement corrélés (avec un coefficient de corrélation de -0,547) sur la période étudiée.

De même, la décomposition des effets de substitution et des effets de lissage sur la variation du solde courant lors d'une détérioration temporaire des termes de 
42

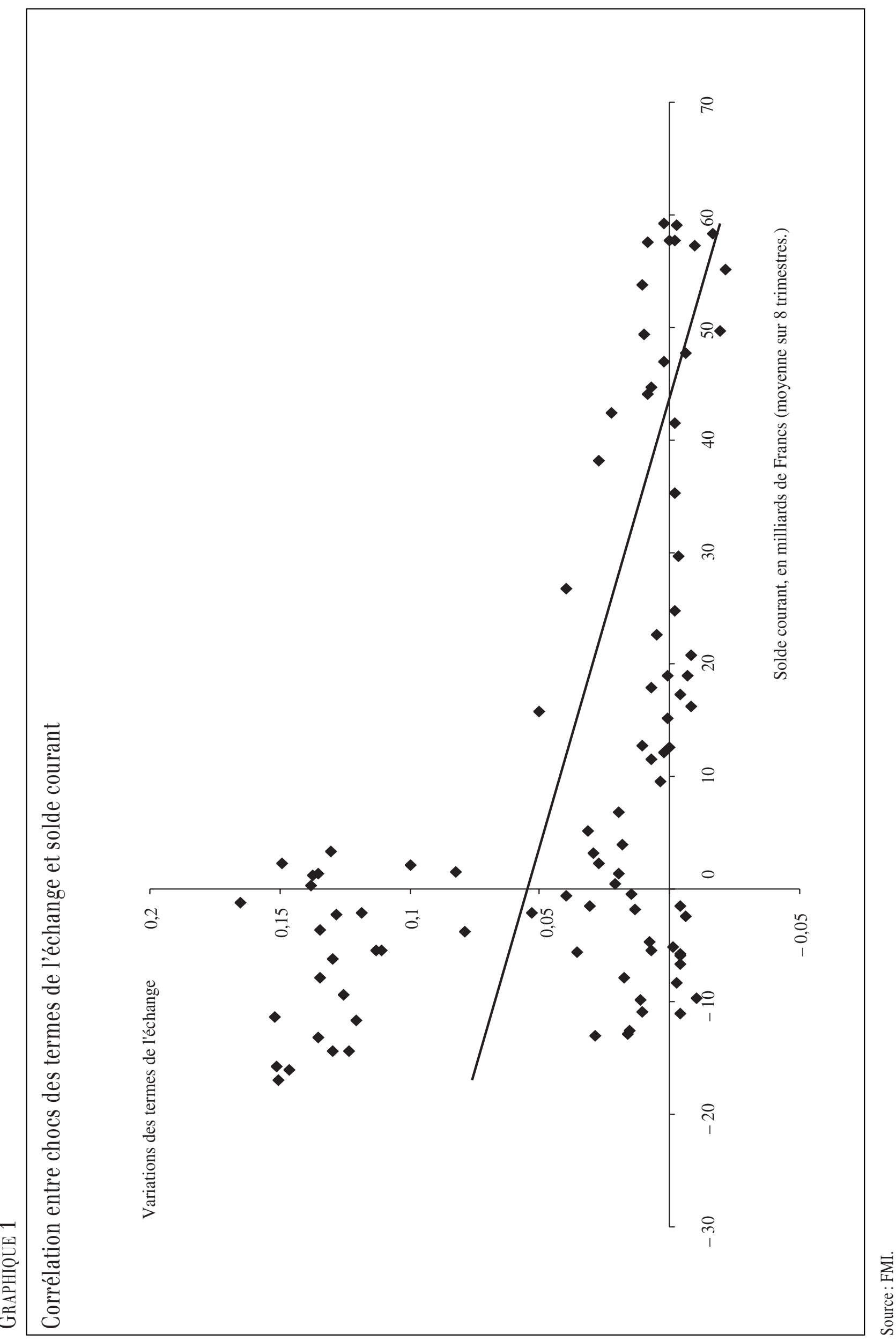

Économie internationale, la revue du CEPII n ${ }^{\circ} 86,2^{\mathrm{E}}$ TRIMESTRE 2001 
l'échange de $1 \%$ (TABLEAU 4) montre que la taille des effets de lissage est plus importante que celle des effets de substitution. Quelle que soit la période considérée, puisque les effets de substitution (intertemporels et intratemporels) ne compensent que partiellement les effets de lissage intertemporel, le compte courant se détériore suite à un choc défavorable des termes de l'échange. L'analyse sur plusieurs sous-périodes montre aussi que l'effet total d'un choc temporaire des termes de l'échange sur le solde extérieur de l'économie française est moins fort depuis le milieu des années quatre-vingt à cause de la diminution de l'élasticité de lissage intertemporel de la consommation. Ce résultat n'a rien de surprenant ici car la facture des importations (notamment énergétiques) liée aux chocs des termes de l'échange a considérablement diminué depuis les premiers chocs pétroliers. Toutefois, dans la mesure où l'effet de perte de pouvoir d'achat sur la demande des ménages français reste toujours plus fort que les effets de substitution, un ralentissement de la demande en France (et de la croissance) est à craindre suite aux modifications récentes des termes de l'échange (consécutives aux hausses du brut et des dépréciations de l'euro).

TABleau 4



\section{$\int$ onclusion}

L'objectif poursuivi dans cet article a été d'estimer les conséquences d'un choc temporaire des termes de l'échange sur la balance courante française. Nous avons alors utilisé un modèle intertemporel de la balance courante qui tient compte des effets de prix relatifs. La modélisation entre biens échangeables et non échangeables permet, en effet, de mesurer directement les paramètres structurels tels que les élasticités de substitution intertemporelles et intratemporelles. Les enseignements tirés de nos résultats rejoignent ceux de Agénor et alii (1999) dans la mesure où l'approche de lissage intertemporel de la balance courante apparaît comme expliquant le mieux les fluctuations des comptes extérieurs français lors des chocs des termes de l'échange. Ce sont, en effet, les effets revenus qui motivent le comportement des agents privés et influencent leur décision d'épargne. En 
rapprochant ces résultats de l'analyse de l'impact de la détérioration récente des termes de l'échange en France, due aux hausses du cours du brut et aux dépréciations successives de l'euro, les estimations obtenues semblent ici montrer que ce choc devrait entraîner une dégradation temporaire du compte courant.

De même, ces résultats semblent confirmer l'argument de Agénor et Bismut (1999) selon lequel l'accumulation récente des surplus de la balance courante en France (depuis 1992-93) ne serait pas due à un retour à une surcompétitivité de l'économie française mais reflète plutôt les perspectives pessimistes des consommateurs français sur leurs revenus futurs. En effet, l'anticipation par les agents français d'une dégradation de leurs revenus permanents liée à la montée de l'endettement public (constituant la promesse des prélèvements futurs) motiverait leur comportement de lissage intertemporel de la consommation (à la baisse) et expliquerait ainsi les surplus récents. La stratégie de désinflation compétitive, qui a été un des objectifs majeur de la politique macroéconomique française depuis 1983 jusqu'à récemment, est alors remise en cause. Elle est supposée conduire à une croissance plus forte grâce à l'amélioration de la compétitivité de l'économie française et au desserrement de la contrainte extérieure. Cependant, la responsabilité de la désinflation compétitive dans les fluctuations du commerce extérieur français n'est pas claire. Certes, cette politique a permis une inflation modérée et un redressement des marges des entreprises françaises. Mais, on ne décèle pas une tendance à la dépréciation réelle du franc (Agénor \& Bismut, 1999) et, au vue de nos simulations, le compte extérieur de la France semble être relativement insensible aux mouvements des prix relatifs ${ }^{15}$. Or, la recherche de l'équilibre externe (et donc le respect de la contrainte extérieure) a constitué pendant longtemps le principal obstacle aux politiques de relance par la demande ${ }^{16}$ et a eu un coût très élevé en terme de chômage.

Enfin, les résultats économétriques trouvés indiquent aussi que l'effet de la réallocation des dépenses de consommation est loin d'être parfait (total). Ce qui implique que le degré de transmission d'une variation des termes de l'échange sur les prix des importations françaises n'est que partiel (Obstfeld, 2000; Obstfeld \& Rogoff, 2000). Une éventuelle segmentation internationale du marché français permet aux producteurs mondiaux de discriminer leur prix. Deux voies de prolongement à cette étude sont alors possibles. Soit, adapter au cas français un modèle intertemporel de la balance courante qui tient compte de la discrimination des prix des biens importables selon le pays d'accueil. Soit, dans le cadre intégré du marché unique européen, adopter une décomposition de la consommation des biens échangeables en France entre les biens importés des pays de l'Union européenne et ceux venants du reste du monde. Cette dernière approche devrait permettre d'estimer la sensibilité du compte courant français par rapport aux variations des prix relatifs intra-européens.

15. Les contributions du taux de change réel et de la position relative dans le cycle aux variations récentes du solde commercial sont faibles par rapport aux facteurs résiduels (Agénor \& Bismut, 1999). Ces derniers peuvent correspondre à des facteurs hors prix (comme la spécialisation intra-branche par exemple).

16. Davantage encore que la recherche de l'équilibre à moyen terme du compte courant, ce sont les recommandations des niveaux d'inflation très bas par la Banque centrale européenne qui constitue sans doute aujourd'hui l'une des causes principales de l'absence de relance économique.

17. L'auteur tient à remercier C. Tavera et J.-C. Poutineau pour leurs conseils et commentaires ainsi que deux rapporteurs anonymes pour leurs remarques et critiques dans la réalisation de cet article. Il reste seul responsable de toutes erreurs ou omissions. 
AnNeXe A.1

SOURCE ET CONSTRUCTION DES DONNÉES

Les données annuelles proviennent de DATASTREAM à partir de différentes bases de données (FMI, OCDE). Les séries suivantes y ont été collectées:

— le taux d'intérêt à 3 mois sur les bons du Trésor américain $(r)$ utilisé comme «proxie » du taux d'intérêt mondial;

- le taux d'intérêt réel $(R)$ formé par le taux d'intérêt nominal sur les bons du Trésor moins le taux de variation du déflateur implicite du PIB (def);

- le PIB nominal aux prix du marché $(Y)$;

- les exportations de biens $(x)$;

- les importations des biens $(m i)$;

- la population ( $p o p)$;

- les déflateurs implicites des importations $(d m)$ et des services $(d c)$;

- les taxes douanières $(d u t)$;

- les PIB dérivées des secteurs agricoles, miniers et manufacturiers ( $\mathrm{amm}$ ) (la production de bien échangeable).

La part des biens de consommation dans le total des marchandises importés $(s)$ a été collectée dans International Trade Statistics Yearbook (ONU).

Ces données ont alors permis de construire les séries suivantes:

- la production domestique des substituts aux importations $d m s=a m m-x$;

- les importations des biens de consommation $m c g=s . m i$;

- la consommation des biens importables par tête d'habitant $m=(m c g+d m s) / p o p$;

- le prix des biens importables $p=d m(1+((d u t / m) / 100))$;

- la consommation des biens non échangeables par tête $n=(y-a m m) / p o p$;

- le prix des biens non échangeables $q=d c$.

ANNEXe A.2

TABleau A.2.1

\begin{tabular}{|c|c|c|c|c|c|}
\hline \multirow[b]{2}{*}{ Tests de type } & \multirow[b]{2}{*}{$p_{t} / q_{t}$} & \multirow[b]{2}{*}{$n_{t} / m_{t}$} & & \multicolumn{2}{|c|}{ Tests de racine unité* } \\
\hline & & & $\ln \left(p_{t} / q_{t}\right)$ & $\ln \left(n_{t} / m_{t}\right)$ & $\hat{e}_{t}$ \\
\hline $\begin{array}{l}\mathrm{ADF} \\
\mathrm{PP}\end{array}$ & $\begin{array}{l}-1,28 \\
-1,21\end{array}$ & $\begin{array}{l}-0,39 \\
-0,09\end{array}$ & $\begin{array}{l}-1,08 \\
-1,11\end{array}$ & $\begin{array}{l}-1,09 \\
-0,87\end{array}$ & $\begin{array}{l}-3,19 * * \\
-3,35 * *\end{array}$ \\
\hline \multicolumn{6}{|c|}{$\begin{array}{l}\text { * Les tests de Dickey-Fuller Augmentés (ADF) et de Phillips-Perron (PP) incluent une constante. } \\
\text { ** Indique que l'hypothèse nulle d'une racine unitaire peut être rejetée à un niveau de risque de } 5 \% \text {. La valeur critique pour } 25 \\
\text { observations est de }-3,00 \text {. }\end{array}$} \\
\hline
\end{tabular}


Tableau A.2.2

\section{Résultats des autres études}

Auteurs

Ostry et Reinhardt

(1992)

Cashin et Mc Dermott (1998)
Échantillon

Pays de trois zones: Afrique, Asie et Amérique latine.

Australie, Canada, Nouvelle-Zélande, Royaume-Uni et États-Unis.
Valeurs des paramètres

$\sigma$ significativement différent de zéro pour tous les pays. $\theta>1$ pour le panel, $\theta<1$ pour l'Asie et l'Amérique latine.

$\sigma$ significativement différent de zéro pour tous les pays sauf pour la Nouvelle-Zélande. $\theta$ non significatif pour les États-Unis et $\theta<1$ pour la Nouvelle-Zélande.

\section{RÉFÉRENCES}

Agénor P.R. \& C. Bismut (1999), « Pourquoi la France accumule-t-elle des surplus de balance courante? », Revue Française d'Économie, 14, juin, p. 61-105.

Agénor P.R., C. Bismut, P. Cashin \& C.J. Mc Dermott (1999), «Consumption Smoothing and The Current Account: Evidence for France, 1970-1996», Journal of international Money and Finance, 18 , p. 1-12.

Amano R.A., W. Ho \& T.S. Wirjanto (1998), « Intraperiod and Intertemporal Substitution in Import Demand », Cahier de Recherche du CREFE, n 84, Université du Québec à Montréal.

Cashin P. \& C.J. Mc Dermott (1998), Terms of Trade Shocks and the Current Account, IMF Working Paper, WP/98/177, décembre.

Cooley T.F. \& M. Ogaki (1996), «A Time Series Analysis of Real Wages, Consumption and Asset Returns », Journal of Applied Econometrics, n 11, p. 119-34.

Gavin M. (1990), « Structural Adjustment to a Terms of Trade Disturbance: The Role of Relative Prices », Journal of International Economics, 28, p. 217-43.

Gosh A.R. (1995), «International Capital Mobility Amongst the Major Industrialized Countries: Too Little or Too Much? », Economic Journal, n 105, p. 107-28.

Hamilton J.D. (1994), Time Series Analysis, Princeton University Press, Princeton.

Hansen L.P. (1982), « Large Sample Properties of Generalised Method of Moments Estimators », Econometrica, 50, p. 47-60.

Obstfeld M. (2000), International Macroeconomics: Beyond the Mundell-Fleming Model, University of California at Berkeley.

Obstfeld M. \& K. Rogoff (1982), « Aggregate Spending and the Terms of Trade: Is there a LaursenMetzler Effect? », Quarterly Journal of Economics, 97, p. 251-70. (Cambridge: Massachussets). (1996), Foundations of International Macroeconomics, MIT Press

(2000), « New Directions for Stochastic Open Economy Models », Journal of International Economics, 50, p. 117-53.

Ogaki M. (1993), « Generalized Method of Moments: Econometric Applications », dans Handbook of Statistics, 11, p. 445-488, Elsevier. 
Ogaki M. \& C. Reinhardt (1998), « Measuring Intertemporal Substitution: The Role of Durable Goods », Journal of Political Economy, n 106, p. 1078-98.

Ostry J.D. (1988), « The Balance of Trade, Terms of Trade, and Real Exchange Rate: An Intertemporel Optimizing Framework», 35, IMF Staff Papers, 35, p. 541-73.

Ostry J.D. \& C. Reinhardt (1992), « Private Saving and Terms of Trade Shocks: Evidence from Developing Countries », IMF Staff Papers, 39, p. 495-517.

Stock J.H. \& M.W. Watson (1993), « A Simple Estimator of Cointegrating Vectors in Higher Order Integrated Systems », Econometrica, 61, juillet, p. 783-820. 